\title{
STATISTICAL ATTITUDE DETERMINATION
}

\author{
F. Landis Markley \\ NASA's Goddard Space Flight Center \\ Greenbelt, MD 20771, USA
}

\section{INTRODUCTION}

All spacecraft require attitude determination at some level of accuracy. This can be a very coarse requirement of tens of degrees, in order to point solar arrays at the sun, or a very fine requirement in the milliarcsecond range, as required by Hubble Space Telescope. A toolbox of attitude determination methods, applicable across this wide range, has been developed over the years [1]. There have been many advances in the thirty years since the publication of Reference [1], but the fundamentals remain the same. One significant change is that onboard attitude determination has largely superseded ground-based attitude determination, due to the greatly increased power of onboard computers. The availability of relatively inexpensive radiation-hardened microprocessors has led to the development of "smart" sensors, with autonomous star trackers being the first spacecraft application. Another new development is attitude determination using interferometry of radio signals from the Global Positioning System (GPS) constellation. This article reviews both the classic material and these newer developments at approximately the level of [1], with emphasis on - methods suitable for use onboard a spacecraft. We discuss both "single frame" methods that are based on measurements taken at a single point in time, and sequential methods that use information about spacecraft dynamics to combine the information from a time series of measurements.

\section{SINGLE FRAME METHODS}

We first consider single-frame methods based on vector measurements. The length of a vector has no information relevant to attitude determination, so each reference vector contains two independent scalar pieces of attitude information. Many spacecraft attitude determination methods use exactly two vector measurements, the minimum required to determine the attitude [2]. Examples are the unit vector to the Sun and the Earth's magnetic field vector for coarse 'sun-mag' attitude determination or unit vectors to two stars for fine attitude determination. TRIAD, the earliest published algorithm for determining spacecraft attitude from two vector measurements, has been widely used in both ground-based and onboard attitude determination $[1,3]$. Since most modern applications use more than two vectors, we will only consider methods based on Wahba's optimality criterion, which optimally weight an arbitrary number (greater than one!) of observations. We will also discuss single-frame methods that convert GPS phase measurements to equivalent vector measurements.

\section{Wahba's Problem}

In 1965, Grace Wahba posed the problem of finding the direction cosine matrix, or attitude matrix, as the orthogonal matrix $A$ with determinant +1 that minimizes the loss function [4]

$$
L(A) \equiv \frac{1}{2} \sum_{i} a_{i}\left|\mathbf{b}_{2}-A \mathbf{r}_{1}\right|^{2},
$$

where $\left\{\mathbf{b}_{i}\right\}$ is a set of unit vectors measured in a spacecraft's body frame, $\left\{\mathbf{r}_{i}\right\}$ are the corresponding unit vectors in a reference frame, and $\left\{a_{i}\right\}$ are non-negative weights. We can choose the weights to be inverse variances, $a_{i}=\sigma_{i}^{-2}$, in order to relate Wahba's problem to Maximum Likelihood Estimation [5]. This choice differs from that of Wahba and many other authors, who assumed the weights normalized to unity, and it has no effect on the attitude estimate. Wahba's loss function is motivated by the fact that in the absence of measurement errors,

$$
\mathbf{b}_{i}=A \mathbf{r}_{i} \text {. }
$$

It is possible and has proven very convenient to write the loss function as

$$
L(A)=\lambda_{0}-\operatorname{tr}\left(A B^{T}\right)
$$

with

$$
\lambda_{0} \equiv \sum_{i} a_{i}
$$

and

$$
B \equiv \sum_{i} a_{i} \mathbf{b}_{i} \mathbf{r}_{i}^{T}
$$


Now it is clear that $L(A)$ is minimized when the trace, $\operatorname{tr}\left(A B^{T}\right)$, is maximized. J. L. Farrell and J. C.

Stuelpnagel , R. H. Wessner, J. R. Velman, J. E. Brock, $R$. Desjardins, and Wahba presented the first solutions of Wahba's problem; but their solutions did not find extensive application [6].

Wahba's problem is closely related to the orthogonal Procrustes problem, which is to find the orthogonal matrix $A$ closest to $B$ in the sense of the Frobenius (or Euclidean, Schur, or Hilbert-Schmidt) norm [7]

$\|M\|_{\mathrm{F}}^{2} \equiv \sum_{t, j} M_{y}^{2}=\operatorname{tr}\left(M M^{T}\right)$.

Since

$\|A-B\|_{\mathrm{F}}^{2}=3+\|B\|_{\mathrm{F}}^{2}-2 \operatorname{tr}\left(A B^{T}\right)$,

Wahba's problem is equivalent to the orthogonal Procrustes problem with the proviso that the determinant of $A$ must be +1 .

\section{Davenport's $q$ Method}

Paul Davenport employed the quaternion parameterization of the attitude matrix, Eq. (31) of Section 5.5.1, to provide the first successful application of Wahba's problem to spacecraft attitude determination $[1,8,9]$. The notation in this article differs from that in Section 5.5.1, though; we use

$$
q=\left[\begin{array}{c}
\mathbf{q} \\
q_{4}
\end{array}\right]=\left[\begin{array}{c}
\mathbf{e} \sin (\phi / 2) \\
\cos (\phi / 2)
\end{array}\right]=\left[\begin{array}{c}
\beta_{1} \\
\beta_{2} \\
\beta_{3} \\
\beta_{0}
\end{array}\right] .
$$

Since $A(q)$ is a homogeneous quadratic function of $q$, the scalar $\operatorname{tr}\left(A B^{T}\right)$ can be written as a quadratic form

$$
\operatorname{tr}\left(A B^{T}\right)=q^{r} K q,
$$

where $K$ is the symmetric traceless matrix

$$
K \equiv\left[\begin{array}{cc}
S-I \operatorname{tr} B & \mathbf{z} \\
\boldsymbol{z}^{T} & \operatorname{tr} B
\end{array}\right],
$$

with $I$ being the $3 \times 3$ identity matrix,

$S \equiv B+B^{T}$,

and $\mathbf{z} \equiv\left[\begin{array}{c}B_{23}-B_{32} \\ B_{31}-B_{13} \\ B_{12}-B_{21}\end{array}\right]=\sum_{t} a_{t} \mathbf{b}_{t} \times \mathbf{r}_{\imath}$.

It is easy to prove that the optimal unit quaternion is the normalized eigenvector of $K$ with the largest eigenvalue, i,e., the solution of

$$
K q_{\mathrm{opt}} \equiv \lambda_{\max } q_{\mathrm{opt}} .
$$

Very robust algorithms exist to solve the symmetric eigenvalue problem $[7,10]$. There is no unique solution if the two largest eigenvalues of $K$ are equal. This is not a failure of the $q$ method; it means that the data aren't sufficient to determine the attitude uniquely.

\section{Quaternion Estimator (QUEST)}

This algorithm, first applied in the MAGSAT mission in 1979, is the most widely used algorithm for Wahba's problem [11]. Equation (13) is equivalent to the two equations

$\left[\left(\lambda_{\max }+\operatorname{tr} B\right) I-S\right] \mathbf{q}=q_{4} \mathrm{z}$

and

$\left(\lambda_{\max }-\operatorname{tr} B\right) q_{4}=\mathbf{q}^{T} \mathbf{z}$

Since the inverse of a matrix is equal to its adjoint (or adjugate) divided by its determinant $[7,10]$, Eq. (14) gives the optimal unit quaternion as

$q_{\mathrm{opt}}=\frac{1}{\sqrt{\gamma^{2}+|\mathrm{x}|^{2}}}\left[\begin{array}{l}\mathrm{x} \\ \gamma\end{array}\right]$,

where

$\mathbf{x} \equiv \operatorname{adj}\left[\left(\lambda_{\max }+\operatorname{tr} B\right) I-S\right] \mathbf{z}$.

and

$\gamma \approx \operatorname{det}\left[\left(\lambda_{\max }+\operatorname{tr} B\right) I-S\right]$.

The optimal quaternion is not defined by Eq. (16) if $\gamma^{2}+|\mathbf{x}|^{2}=0$, so Shuster devised the method of sequential rotations to handle this case [11, 12]. This computes the quaternion in a rotated coordinate frame, using rotations that are very easily computed.

Equations (16)-(18) clearly require knowledge of $\lambda_{\max }$, which is obtained by substituting these equations into Eq. (15), yielding 


$$
\begin{aligned}
0 & =\psi\left(\lambda_{\max }\right) \equiv \gamma\left(\lambda_{\max }-\operatorname{tr} B\right)-\mathbf{x}^{T} \mathbf{z} \\
& =\left(\lambda_{\max }^{2}-a\right)\left(\lambda_{\max }^{2}-b\right)-c\left(\lambda_{\max }-\operatorname{tr} B\right)-\mathbf{z}^{T} S^{2} \mathbf{z}
\end{aligned}
$$

where

$$
\begin{aligned}
& a \equiv(\operatorname{tr} B)^{2}-\operatorname{tr}(\operatorname{adj} S), \\
& b \equiv(\operatorname{tr} B)^{2}+\mathbf{z}^{T} \mathbf{z}, \\
& c \equiv \operatorname{det} S+\mathbf{z}^{T} S \mathbf{z} .
\end{aligned}
$$

Equation (19) is simply the characteristic equation $\operatorname{det}\left(\lambda_{\max } I-K\right)=0$. Shuster observed that $\lambda_{\max }$ is very close to $\lambda_{0}$ since the optimized loss function

$$
L\left(A_{\mathrm{opt}}\right)=\lambda_{\mathrm{o}}-\lambda_{\max }
$$

is expected to be small. Thus $\lambda_{\max }$ can be easily obtained by Newton-Raphson iteration of Eq. (19), starting from $\lambda_{3}$ as the initial estimate. In fact, a single iteration is generally sufficient. The analytic solution of the quartic characteristic equation is slower and no more accurate than the iterative solution. Numerical analysts know that solving the characteristic equation for $\lambda_{\max }$ makes QUEST less robust in principle than Davenport's original $q$ method, but it is quite reliable in practice if the characteristic equation is evaluated in the partially-factored form of Eq. (19) [13].

\section{Singular Value Decomposition Method}

The Singular Value Decomposition (SVD) of $B$ is [7]

$$
B=U \Sigma V^{T}=U\left(\operatorname{diag}\left[\begin{array}{lll}
\Sigma_{11} & \Sigma_{22} & \Sigma_{33}
\end{array}\right]\right) V^{T},
$$

where $U$ and $V$ are orthogonal, and the singular values obey the inequalities $\Sigma_{11} \geq \Sigma_{22} \geq \Sigma_{33} \geq 0$. The trace is invariant under cyclic permutation of its argument, so

$$
\operatorname{tr}\left(A B^{T}\right)=\operatorname{tr}\left(U^{T} A V \operatorname{diag}\left[\begin{array}{lll}
\Sigma_{11} & \Sigma_{22} & \Sigma_{33}
\end{array}\right]\right) .
$$

The trace is maximized, consistent with the constraint $\operatorname{det} A=1$, by the optimal attitude matrix [14]:

$$
A_{\mathrm{opt}}=U \operatorname{diag}\left[\begin{array}{lll}
1 & 1 & (\operatorname{det} U)(\operatorname{det} V)] V^{T} .
\end{array}\right.
$$

The SVD solution is completely equivalent to the original solution by Farrell and Stuelpnagel, but robust SVD algorithms are readily available now $[7,10]$. In fact, computing the SVD is one of the most robust numerical algorithms.

\section{Fast Optimal Attitude Matrix (FOAM)}

The SVD decomposition of $B$ gives a convenient representation for $\operatorname{adj} B, \operatorname{det} B$, and $\|B\|_{\mathrm{F}}^{2}$. These can be used to write the optimal attitude matrix as [15]

$$
A_{\text {opt }}=\xi^{-1}\left[\left(\kappa+\|B\|_{F}^{2}\right) B+\lambda_{\max } \operatorname{adj} B^{T}-B B^{T} B\right],
$$

where

$$
\kappa \equiv \frac{1}{2}\left(\lambda_{\max }^{2}-\|B\|_{F}^{2}\right)
$$

and

$$
\zeta=\kappa \lambda_{\max }-\operatorname{det} B .
$$

All the quantities in Eqs. (25)-(27) can be computed without performing the SVD of $B$. In this method, $\lambda_{\max }$ is found by substituting Eq. (25) into

$$
\lambda_{\max }=\operatorname{tr}\left(A_{\mathrm{opt}} B^{T}\right)
$$

which gives, after some matrix algebra,

$$
\begin{aligned}
0 & =\psi\left(\lambda_{\max }\right) \\
& \equiv\left(\lambda_{\max }^{2}-\|B\|_{\mathrm{F}}^{2}\right)^{2}-8 \lambda_{\max } \operatorname{det} B-4\|\operatorname{adj} B\|_{\mathrm{F}}^{2} .
\end{aligned}
$$

Equations (19) and (29) for $\psi\left(\lambda_{\max }\right)$ would be numerically identical with infinite-precision computations.

\section{Estimator of the Optimal Quaternion}

Davenport's eigenvalue equation, Eq. (13), says that the optimal quaternion is orthogonal to all the columns of the matrix $K-\lambda_{\text {max }} I$. Thus $q_{\mathrm{opt}}$ can be computed by normalizing any non-zero column of $\operatorname{adj}\left(K-\lambda_{\max } I\right)$. Let $F$ denote the symmetric $3 \times 3$ matrix obtained by deleting the $k$ th row and $k$ th column from $K-\lambda_{\max } I$, and let $\mathbf{f}$ denote the three-component column vector obtained by deleting the $k$ th element from the $k$ th column of $K-\lambda_{\max } I$. Then the $k$ th element of the optimal quaternion is given by

$$
\left(q_{\mathrm{opt}}\right)_{k}=-d \operatorname{det} F \text {, }
$$

and the other three elements are .

$$
\left(q_{\mathrm{opt}}\right)_{1, \ldots, k-1, k+1, \ldots, 4}=d(\operatorname{adj} F) \mathbf{f},
$$

where the scalar $d$ is determined by normalization of the quaternion [16, 17]. It is desirable to choose $k$ to index the column with the maximum Euclidean norm. 
Because $K$ is symmetric, it is only necessary to examine the diagonal elements of the adjoint to determine which column to use. This method is the First EStimator of the Optimal Quaternion (ESOQ).

A second EStimator of the Optimal Quaternion (ESOQ2) is based on the rotation axis and angle [18]. Inserting Eq. (8) into Eqs. (14) and (15) gives

$z \cos (\phi / 2)=\left[\left(\lambda_{\max }+\operatorname{tr} B\right) I-S\right] \mathrm{esin}(\phi / 2)$

and

$$
\left(\lambda_{\max }-\operatorname{tr} B\right) \cos (\phi / 2)=\mathbf{z}^{T} \mathbf{e} \sin (\phi / 2) .
$$

Multiplying Eq. (32) by $\lambda_{\max }-\operatorname{tr} B$ and substituting Eq. (33) gives

$$
M e \sin (\phi / 2)=0 \text {, }
$$

where $M$ is the $3 \times 3$ matrix

$$
M=\left(\lambda_{\max }-\operatorname{tr} B\right)\left[\left(\lambda_{\max }+\operatorname{tr} B\right) I-S\right]-\mathbf{z z} \mathbf{z}^{\mathrm{T}} .
$$

These computations lose numerical significance if $\lambda_{\max }-\operatorname{tr} B$ and $\mathrm{z}$ are near zero, which would be the case for zero rotation angle. We can always avoid this singular condition by rotating the reference system to ensure that $\operatorname{tr} B$ is less than or equal to zero. Then Eq. (34) says that the rotation axis is a null vector of $M$.

The columns of adj $M$ are the cross products of the columns of $M$. Because $M$ is singular, all these columns are parallel, and all are parallel to the rotation axis $\mathbf{e}$. Thus we set

$$
\mathbf{e}=\mathbf{y} /|\mathbf{y}|,
$$

where $y$ is the column of adj $M$ (i.e., the cross product) with maximum norm. It is only necessary to examine the diagonal elements of the adjoint matrix to determine which column to use. The rotation angle is found from Eq. (33) or one of the components of Eq. (32). The use of a rotated reference system to ensure a non-positive $\operatorname{tr} B$ makes Eq. (33) the best choice. With Eq. (36), this can be written

$$
\left(\lambda_{\max }-\operatorname{tr} B\right)|\mathbf{y}| \cos (\phi / 2)=(\mathbf{z} \cdot \mathbf{y}) \sin (\phi / 2),
$$

which means that there is some scalar $h$ for which

$$
\cos (\phi / 2)=h(z \cdot y)
$$

and

$$
\sin (\phi / 2)=h\left(\lambda_{\max }-\operatorname{tr} B\right)|y| .
$$

Substituting into Eq. (8) gives the optimal unit quaternion as

$$
q_{\mathrm{opt}}=\frac{1}{\sqrt{\left.\left(\lambda_{\max }-\operatorname{tr} B\right) \mathbf{y}\right|^{2}+(\mathbf{z} \cdot \mathbf{y})^{2}}}\left|\begin{array}{c}
\left(\lambda_{\max }-\operatorname{tr} B\right) \mathbf{y} \\
\mathbf{z} \cdot \mathbf{y}
\end{array}\right| .
$$

Note that it is not necessary to normalize the rotation axis. ESOQ2 does not define the rotation axis uniquely if $M$ has rank less than two. This includes the case of unobservable attitude and also the case of zero rotation angle. Requiring $\operatorname{tr} B$ to be non-positive avoids the zero rotation angle singularity, however.

\section{Comparison of Algorithms for Wahba's Problem}

Extensive tests have shown that all these methods are capable of providing equal accuracy $[13,19,20]$. Davenport's $q$ method and the SVD method are significantly slower than the other methods, with the $q$ method being somewhat faster except in the least interesting two-observation case. The fastest of the other algorithms, QUEST, ESOQ and ESOQ2, are nearly equal in speed. The FOAM method has intermediate speed.

The $q$ and SVD methods are the most robust, reliable, and accurate estimators, since they are based on welltested general-purpose matrix algorithms. The faster methods are less robust in principle, since they solve the quartic characteristic polynomial equation for the maximum eigenvalue, a procedure that is potentially numerically unreliable. They perform as well as the more robust algorithms in practice, though.

\section{Single-Frame GPS Attitude Determination}

The use of GPS for satellite navigation is widespread; a user can compute the three Cartesian components of his position and his clock bias from the transit times of signals received from no fewer than four satellites of the Global Positioning System (GPS) constellation. Attitude determination using phase differences of GPS signals received by antennas located at different locations on the spacecraft is a later development.

Pairs of GPS antennas form a set of $n \geq 2$ baselines $\left\{\mathbf{b}_{i}\right\}$ in the spacecraft body frame. The $m \geq 4$ unit vector sightlines $\left\{\mathbf{s}_{j}\right\}$ from the user spacecraft to the GPS satellites can be computed from the GPS position solution. The different path lengths from each GPS satellite to the antennas at the two ends of each baseline create $m n$ phase differences of the received signals

$\phi_{y}^{i \text { ine }}=2 \pi\left(n_{y j}^{\phi}+\mathbf{b}_{I}^{T} A \mathbf{s}_{j} / \lambda\right)$, 
where the integers $n_{i j}^{\phi}$ are phase ambiguities, $A$ is the attitude matrix, and $\lambda$ is the wavelength of the GPS signal. After the integer phase ambiguities have been found using one of several algorithms $[21,22]$, we compute the normalized measurements

$$
z_{i j} \equiv \lambda\left(\phi_{i j}^{\text {measured }}-2 \pi n_{i j}^{\phi}\right) \text {. }
$$

The optimal attitude solution is the attitude matrix minimizing the loss function.

$$
L_{\mathrm{GPS}}(A) \equiv \frac{1}{2} \sum_{y} a_{y j}\left(z_{y}-\mathbf{b}_{1}^{T} A \mathbf{s}_{\jmath}\right)^{2},
$$

for some weights $a_{i j}$. This is similar to Wahba's loss function, but it is not as easy to solve. Solutions can be found with difficulty, however [23].

If we knew the representations of the baseline vectors in the reference frame, which we denote by $\left\{\mathbf{r}_{i}\right\}$ as in Wahba's problem, we could use one of the algorithms for solving that problem to compute the attitude matrix. In view of Eq. (2), we compute these as the $\mathbf{r}_{i}$ minimizing the loss functions

$$
L_{i}\left(\mathbf{r}_{i}\right) \equiv \frac{1}{2} \sum_{j} a_{i j}\left(z_{l y}-\mathbf{r}_{i}^{T} \mathbf{s}_{j}\right)^{2},
$$

for $i=1,2, \ldots, n[24]$. The minimization gives

$\mathbf{r}_{i}=S_{1}^{-1}\left[\sum, a_{y j} z_{y j} \mathbf{s}_{j}\right]$,

where

$$
s_{t} \equiv \sum_{j} a_{y j} s_{j} s_{j}^{T} \text {. }
$$

These solutions only exist if the matrices $S_{\text {, }}$ all have rank three, which requires that the sightlines $s_{j}$ not be coplanar, as will generally be the case. The computational burden is reduced if the weights $a_{i j}$ are independent of the baseline label $i$, since then all the $S_{i}$ will be equal, and only one matrix must to be computed and inverted for each set of sightlines.

The computational burden would also be reduced if we reversed the roles of the baselines and sightlines, since the matrix corresponding to $S_{i}$ would depend on the baselines in the body frame, which are constant. This requires at least three non-coplanar baselines, however, which are not always available.

This method may give representations of the observed and computed vectors in the reference and body frames that have different lengths, but they should not differ greatly if measurement errors are small. The constraint of equal lengths in the two frames can be used to find a solution if a matrix $S_{i}$, or the corresponding matrix using baselines, has rank two $[24,25]$.
The final step of this method is to solve Wahba's problem for the vector sets $\left\{\mathbf{b}_{i}\right\}$ and $\left\{\mathbf{r}_{i}\right\}$; the fact that they are not unit vectors is not important. There are several options for choosing the weights $a_{i}$ in Wahba's loss function, but no choice produces an optimal minimum of the loss function of Eq. (43), in general. The estimates are nearly optimal unless the sightlines are nearly coplanar, though; and simulations show that the computational advantages of this method do not entail a significant loss of accuracy [24].

\section{SEQUENTIAL METHODS}

When observations are acquired over a range of times, it is of ten convenient to employ a filter to propagate past attitude information to the current time and then add information from current measurements. This generally provides more accurate attitude solutions than are obtainable from single-frame estimators, and can also provide estimates when insufficient data are available at a single time for single-frame estimators to provide any solution at all. A prominent example of the latter is magnetometer-only attitude determination [26]. Sequential methods require some knowledge of system dynamics for the time propagation. This can be computed from rotational dynamics, e.g. from Euler's dynamical equations, or obtained from gyro-sensed rates.

The most commonly employed sequential method is the Kalman Filter [27], or more precisely, the extended Kalman filter (EKF) $[28,29]$, since the spacecraft attitude estimation problem is nonlinear. A pplications of the EKF to spacecraft attitude determination up to 1982 have been surveyed in the literature [30]. Several different attitude parameterizations have been used, with the earliest being the Euler angles; but the quaternion has become the most common parameterization [30]. We will discuss only the quaternion parameterization of the EKF, but we also discuss sequential methods related to Wahba's problem that are not EKFs.

\section{Additive Quaternion EKF}

For Kalman filtering, we need the time propagation equations and the measurement update equations for the state vector estimate and its covariance. The state vector will generally include components in addition to the quaternion, but we will ignore these in order to emphasize the attitude representation. We consider the time propagation first. 
Quaternion dynamics are given in our notation by

$\dot{q}=\frac{1}{2}\left[\begin{array}{c}m \\ 0\end{array}\right] \otimes q$

which is a convenient way of writing Eq. (35) of Section 5.5 .1 in terms of the quaternion product defined by

$$
p \otimes q=\left[\begin{array}{c}
\mathbf{p} \\
p_{4}
\end{array}\right] \otimes\left[\begin{array}{c}
\mathbf{q} \\
q_{4}
\end{array}\right] \equiv\left[\begin{array}{c}
p_{4} \mathbf{q}+q_{4} \mathbf{p}-\mathbf{p} \times \mathbf{q} \\
p_{4} q_{4}-\mathbf{p} \cdot \mathbf{q}
\end{array}\right] .
$$

Equation (47) for the quaternion kinematics is exact, by the definition of the angular velocity vector, so no additive process noise appears in this equation; process noise can only enter through $\boldsymbol{\omega}$.

For the additive filter, we write the quaternion and rate errors as additive errors

$q=\hat{q}+\Delta q$

and

$\boldsymbol{\omega}=\hat{\omega}+\Delta \omega$,

where the carets indicate estimates, which are expected values in the additive EKF.

The quaternion error dynamics are found by inserting Eqs. (49) and (50) into Eq. (47), equating zeroth and first order terms in the errors, and ignoring second order terms, giving

$\dot{\hat{q}}=\frac{1}{2}\left[\begin{array}{c}\hat{\omega} \\ 0\end{array}\right] \otimes \hat{q}$

and

$\Delta \dot{q}=\frac{1}{2}\left[\begin{array}{c}\hat{\omega} \\ 0\end{array}\right] \otimes \Delta q+\frac{1}{2}\left[\begin{array}{c}\Delta \omega \\ 0\end{array}\right] \otimes \hat{q}$.

Equation (51) can be written in matrix form as [1]

$\dot{\hat{q}}=\frac{1}{2} \Omega(\hat{\omega}) \hat{q}$,

where

$$
\Omega(\omega) \equiv\left[\begin{array}{cc}
-[\omega \times] & \omega \\
-\omega^{T} & 0
\end{array}\right]
$$

and $[\omega \times]$ is the cross product matrix

$$
[\omega x] \equiv\left[\begin{array}{ccc}
0 & -\omega_{3} & \omega_{2} \\
\omega_{3} & 0 & -\omega_{1} \\
-\omega_{2} & \omega_{1} & 0
\end{array}\right] .
$$

The matrix $\Omega(\omega)$ is skew-symmetric, so Eqs. (47) and (53) preserve the norms of $q$ and $\hat{q}$. Equation (53) has the solution

$\hat{q}(t)=\Phi_{4 \times 4}\left(t, t_{k}\right) \hat{q}\left(t_{k}\right)$,

where $\Phi_{4 \times 4}\left(t, t_{k}\right)$ is the orthogonal quaternion state transition matrix that obeys the differential equation

$$
\dot{\Phi}_{4 \times 4}\left(t, t_{k}\right)=\frac{1}{2} \Omega(\hat{\omega}(t)) \Phi_{4 \times 4}\left(t, t_{k}\right),
$$

with the initial condition that $\Phi_{4 \times 4}\left(t_{k}, t_{k}\right)$ be the $4 \times 4$ identity matrix. The solution of Eq. (52),

$$
\begin{aligned}
\Delta q(t) & =\Phi_{4 \times 4}\left(t, t_{k}\right) \Delta q\left(t_{k}\right) \\
& +\frac{1}{2} \int_{t_{k}}^{i} \Phi_{4 \times 4}\left(t, t^{\prime}\right)\left[\begin{array}{c}
\Delta \omega\left(t^{\prime}\right) \\
0
\end{array}\right] \otimes \hat{q}\left(t^{\prime}\right) \mathrm{d} t^{\prime},
\end{aligned}
$$

is used to find the time propagation of the covariance of the four-component quaternion

$$
P_{4 \times 4} \equiv E\left\{(\Delta q)(\Delta q)^{\mathrm{T}}\right\} \text {, }
$$

where $E$ denotes the expected value.

Instead of continuing blindly with the development of the additive quaternion EKF, we will discuss three problems with this approach [30-32].

The first problem is a conceptual one. The expected value of $q$ cannot be a unit quaternion, because restricting the probability distribution in quaternion space to the surface of a unit sphere means that its expected value must be inside the sphere. It is true that the norm of $\hat{q}$ only differs from unity by second order in $\Delta q$, and that the EKF is inherently a first-order method; but we would like our optimal estimate to be a properly normalized quaternion.

The second problem is computational and is related to the first one. If the quaternion errors are small, they lie approximately in the plane tangent to the quaternion sphere at the expected value, which means that they are nearly orthogonal to the expected value, or algebraically,

$$
\Delta q^{\mathrm{T}} \hat{q} \approx 0
$$


This equation together with $\mathrm{Eq}$. (59) implies that $\hat{q}$ is an eigenvector of the covariance matrix with a very small eigenvalue, i.e. that the covariance is nearly singular [30]. This is acceptable in principle, but numerical errors can lead to a negative eigenvalue, losing positive semidefiniteness.

The most direct response to the normalization question is brute force normalization of the quaternion, which only changes the update to second order, and is therefore outside the purview of the EKF. Other approaches employ pseudo-measurements of the quaternion or of its norm. None of these modifications addresses the covariance singularity question $[31,32]$.

A totally different approach is to relax the requirement of quaternion normalization by letting

$$
A=|q|^{-2}\left\{\left(q_{4}^{2}-|\mathbf{q}|^{2}\right) I+2 \mathbf{q q}^{\mathrm{T}}-2 q_{4}[\mathbf{q} \times \mid\}\right. \text {, }
$$

which is an orthogonal matrix for any $q$. This avoids both the normalization and zero covariance problems; the covariance is nonsingular because the norm of $q$ is not known exactly. This approach implicitly carries the quaternion norm as an additional, unobservable degree of freedom, which is undesirable.

The theoretically ideal solution would be some sort of optimization procedure that constrains the estimate to the non-Euclidean manifold of the rotation group, or equivalently to the unit sphere in quaternion space. A preliminary attempt in this direction has not led to a useful algorithm, however [33].

None of these alternatives addresses the third problem, which is that the additive EKF is inefficient. There is no reason to compute $4 \times 4$ covariance and state transition matrices when an alternative employing $3 \times 3$ matrices is available. We now turn to this alternative, which has come to be known as the multiplicative quaternion extended Kalman filter, or MEKF.

\section{Multiplicative Quaternion EKF (MEKF)}

This algorithm was developed in 1969 [34], and has been used in NASA programs since 1978 [35]; our discussion is based on $[30,36]$. The MEKF retains Eqs. (50), but replaces Eq. (49) by

$q=\hat{\alpha} q \otimes \hat{q}$.

The estimation error is represented by a multiplicative quaternion rather than an additive one, accounting for the name of the algorithm. The dynamics of $\hat{q}$ is defined by Eq. (51), rather than from the expected value of $q$. This gives the dynamic behavior we expect of the estimate, and allows $\hat{q}$ to be normalized exactly, rather than only to first order. In the MEKF $\hat{q}$ equals the expected value only to first order in the errors, which is consistent with the linear EKF approximation.

Equation (62) shows that $\delta q$ is a unit quaternion, also. The EKF implicitly assumes small estimation errors, so $\delta q$ is close to the identity quaternion. Although the rotation group has no global, continuous, nonsingular, three-dimensional parameterization [37]; it has plenty of local, continuous, nonsingular, three-dimensional parameterizations [38]. We will use one of these to parameterize $\delta q$. Since the state vector in a Kalman filter is conventionally designated by $x$, we will use a three-vector $\mathbf{x}$ to parameterize $\delta q$.

We consider four parameterizations. The first takes $\mathbf{x}$ to be the rotation vector $\phi=\phi \mathbf{e}$, so from Eq. (8),

$$
\delta q(\mathbf{x})=\left[\begin{array}{c}
(\mathbf{x} /|\mathbf{x}|) \sin (|\mathbf{x}| / 2) \\
\cos (|\mathbf{x}| / 2)
\end{array}\right] .
$$

This has the advantage that the covariance includes the angular variances in radians ${ }^{2}$, but it is numerically inconvenient. A special form, such as a Taylor series, must be used near the singularity at $\mathbf{x}=0$.

We can retain the interpretation of the covariance matrix in the small angle approximation by setting $\mathbf{x}$ to twice the vector part of $\delta q$, as in [30];

$\delta q(\mathbf{x})=\left[\frac{\mathbf{x} / 2}{\sqrt{1-|\mathbf{x} / 2|^{2}}}\right]$.

A better parameterization is to set $\mathbf{x}$ equal to twice the Gibbs vector $\delta q / \delta q_{4}$, giving

$$
\left.\delta q(\mathbf{x})=\frac{1}{\sqrt{4+|\mathbf{x}|^{2}}} \mid \begin{array}{l}
\mathbf{x} \\
2
\end{array}\right] .
$$

This is equivalent to using a first-order approximation in $\mathbf{X}$ and then normalizing the quaternion, which is useful for minimizing roundoff errors.

As a fourth alternative, $\mathbf{x}$ can be four times the vector of modified Rodrigues parameters $\delta \mathbf{q} /\left(1+\delta q_{4}\right)$, giving

$$
\delta q(\mathrm{x})=\frac{1}{16+|\mathbf{x}|^{2}}\left[\begin{array}{c}
8 \mathrm{x} \\
16-|\mathbf{x}|^{2}
\end{array}\right]
$$

This parameterization has the computational advantage of not requiring any transcendental functions. 
These definitions of $\mathbf{x}$ are equivalent for an EKF, since they have the same first-order approximation,

$\delta q(\mathbf{x}) \approx\left[\begin{array}{c}\mathbf{x} / 2 \\ 1\end{array}\right]$

but they all differ in higher orders.

At this point, the MEKF is treated as a conventional EKF for the parameter $x$, so we need to develop equations for the time propagation of its expectation and covariance. Substituting Eqs. (47) and (51) into the time derivative of Eq. (62) gives

$\frac{1}{2}\left[\begin{array}{c}\omega \\ 0\end{array}\right] \otimes q=\delta \dot{q} \otimes \hat{q}+\frac{1}{2} \delta q \otimes\left[\begin{array}{c}\hat{\boldsymbol{\omega}} \\ 0\end{array}\right] \otimes \hat{q}$.

Substituting Eq. (62) on the left side and cancelling the common factor $\hat{q}$ from all terms then leads to

$\delta \dot{q}=\frac{1}{2}\left(\left[\begin{array}{c}\boldsymbol{\omega} \\ 0\end{array}\right] \otimes \delta q-\delta q \otimes\left[\begin{array}{c}\hat{\boldsymbol{\omega}} \\ \mathbf{0}\end{array}\right]\right)$.

Substituting Eq. (64) into Eq. (66) and using Eq. (48) gives the vector part, to first order in $\mathbf{x}$ and $\Delta \omega$, as

$\dot{\mathbf{x}}=-\hat{\boldsymbol{\omega}} \times \mathbf{x}+\Delta \omega$.

The scalar part of Eq. (66) is trivially satisfied to first order. Equation (67) has the solution

$\mathbf{x}(t)=\Phi_{3 \times 3}\left(t, t_{k}\right) \mathbf{x}\left(t_{k}\right)+\int_{t_{k}}^{t} \Phi_{3 \times 3}\left(t, t^{\prime}\right) \Delta \omega\left(t^{\prime}\right) \mathrm{d} t^{\prime}$,

where $\Phi_{3 \times 3}\left(t, t_{k}\right)$ is the solution of

$\dot{\Phi}_{3 \times 3}\left(t, t_{k}\right)=-[\hat{\omega}(t) \times] \Phi_{3 \times 3}\left(t, t_{k}\right)$,

with the initial condition that $\Phi_{3 \times 3}\left(t, t_{k}\right)$ be the $3 \times 3$ identity matrix. Equation (69) has the same form as the kinematic equation for the attitude matrix, Eq. (11) of Section 5.5.1, so the $3 \times 3$ error state transition matrix can be simply computed by

$$
\Phi_{3 \times 3}\left(t, t_{k}\right)=\hat{A}(t) \hat{A}^{T}\left(t_{k}\right),
$$

where Eq. (31) of Section 5.5.1 and the notation of Eq. (8) give $A(t)$ as a function of $\hat{q}(t)$. Thus no separate integration of Eq. (69) is required.

Equation (68) and the assumption that $\Delta \omega$ has zero mean give the propagation of the expected value of $\mathbf{x}$ as

$\hat{\mathbf{x}}(t)=\Phi_{3 \times 3}\left(t, t_{k}\right) \hat{\mathbf{x}}\left(t_{k}++\right)$, where $\hat{\mathbf{x}}\left(t_{k}++\right)$ is its value after a measurement update at time $t_{k}$ and a reset operation described below. The reset sets $\hat{\mathbf{x}}\left(t_{k}++\right)$ to zero, so $\hat{\mathbf{x}}(t)$ is identically zero between measurements and there is no need to propagate it. The quaternion attitude estimate is propagated by $\mathrm{Eq}$. (56), and $\hat{\mathbf{x}}$ takes a nonzero value only at times when measurements are being processed.

Equation (67) gives the propagation of the three-vector error state $\Delta \mathbf{x}=\mathbf{x}-\hat{\mathbf{x}}$ as

$$
\Delta \mathbf{x}(t)=\Phi_{3 \times 3}\left(t, t_{k}\right) \Delta \mathbf{x}\left(t_{k}\right)+\int_{t_{k}}^{\prime} \Phi_{3 \times 3}\left(t, t^{\prime}\right) \Delta \omega\left(t^{\prime}\right) \mathrm{d} t^{\prime},
$$

which is simpler than Eq. (58) in the additive EKF. This is used to propagate of the $3 \times 3$ covariance

$$
P_{3 \times 3} \equiv E\left\{(\Delta \mathbf{x})(\Delta \mathbf{x})^{T}\right\}
$$

following standard EKF practice $[28,29]$. If the EKF state vector contains components other than attitude errors, these must be propagated in the usual manner, also. Details can be found in the literature $[30,35,36]$.

\section{Measurement Model and Update}

One of the benefits of the EKF is its generality; it is not restricted to vector measurements, but can handle a wide variety of measurement types. This includes horizon crossings from scanning earth sensors, GPS phase measurements, and the horizontal or vertical position of an object detected in the focal plane of a star tracker or digital sun sensor, for example. It can be advantageous to convert measurements to unit vectors to provide a standard interface [39]. This may require a suboptimal representation of sensor measurement errors, but this is seldom important in practice.

Consider a scalar measurement for simplicity, since a vector measurement can be built up from its scalar components. The measurement is modeled as a scalar function $\chi$ of $a$ vector $b$ in the spacecraft body frame, with white noise added.

$z=\chi(\mathbf{b})+$ white measurement noise.

Different measurements will have different $\chi$ and $\mathbf{b}$, in general, but we omit all distinguishing labels for notational convenience. The representation of $\mathbf{b}$ in the body frame is the mapping of its representation $r$ in the reference frame by the attitude matrix:

$\mathbf{b}=A(q) \mathbf{r}=A(\delta q \otimes \hat{q}) \mathbf{r}=A(\delta q) A(\hat{q}) \mathbf{r}=A(\delta q) \hat{\mathbf{b}}$,

where $\hat{\mathbf{b}} \equiv A(\hat{q}) \mathbf{r}$ is the vector in the body frame that is predicted by the estimated quaternion. 
Equation (64) gives the first-order approximation to the attitude matrix

$$
A(\delta q)=I-[\mathrm{x} \times] .
$$

Substituting this into Eqs. (74) and (75) gives, to first order in $\mathbf{x}$,

$$
\begin{aligned}
\chi(\mathbf{b})=\chi(\hat{\mathbf{b}}-\mathbf{x} \times \hat{\mathbf{b}}) & =\chi(\hat{\mathbf{b}})-(\nabla \chi) \cdot(\mathbf{x} \times \hat{\mathbf{b}}) \\
& =\chi(\hat{\mathbf{b}})+(\nabla \chi \times \hat{\mathbf{b}}) \cdot \mathbf{x},
\end{aligned}
$$

where $\nabla \chi$ is the gradient of the measurement function with respect to its argument. Thus the sensitivity of the scalar measurement $z$ with respect to $\mathbf{X}$ is the row vector

$$
H \equiv \partial \chi / \partial \mathbf{x}=(\nabla \chi \times \hat{\mathbf{b}})^{T} .
$$

This equation has an interesting interpretation for an imaging sensor. If $z$ is the horizontal displacement of an image in a focal plane, then $\nabla \chi$ is in the horizontal direction. If $\hat{b}$ is perpendicular to the focal plane, which is approximately true for a sensor with a narrow field of view, then $H$ is in the vertical direction in the focal plane, which is the axis of a rotation that would cause a horizontal displacement of the image.

The computation of the Kalman gain $K$ and the covariance update follow standard EKF practice [28, 29]. The state update is given by

$$
\begin{aligned}
\hat{\mathbf{x}}\left(t_{k}+\right) & =\hat{\mathbf{x}}\left(t_{k}-\right)+K(z-\hat{z}) \\
& =\hat{\mathbf{x}}\left(t_{k}-\right)+K\left[z-\chi(\hat{\mathbf{b}})-H \hat{\mathbf{x}}\left(t_{k}-\right)\right],
\end{aligned}
$$

where $z$ denotes the measured value.

A reset operation is performed after processing all the measurements taken at one time. This operation uses one of Eqs. (63) to reset the quaternion estimate by

$$
\hat{q}\left(t_{k}+\right)=\delta q\left(\left(\hat{\mathbf{x}}\left(t_{k}+\right)\right) \otimes \hat{q}\left(t_{k}-\right),\right.
$$

while simultaneously setting the expected value of the attitude error $\hat{\mathbf{x}}\left(t_{k}++\right)$ to zero, thereby moving the measurement information from $\hat{\mathbf{x}}$ into $\hat{q}$.

Resets can be performed after each measurement update, in which case the term $H \hat{\mathbf{x}}\left(t_{k}-\right)$ in Eq. (79) is always zero; but the reset is usually delayed for computational efficiency until all the updates for a set of simultaneous measurements have been performed. It is imperative to perform a reset before beginning the next time propagation, however, to avoid the necessity of propagating $\mathbf{x}(t)$ between measurements.

The operation of the MEKF is much more straightforward than indicated by this description, which has attempted to explain and motivate as well as to describe the algorithm.

\section{Quaternion Measurement Models}

A modern star tracker may track between 5 and 50 stars simultaneously, match them to stars in an internal star catalogue, and compute its attitude as an inertiallyreferenced quaternion using one of the single frame methods discussed above. The computation can also estimate the covariance of the attitude error angle vector $[11,14,15]$. It is a simple matter to transform these quantities from the star tracker reference frame to the spacecraft frame to produce a quaternion observation $q_{o b s}$ and a $3 \times 3$ measurement covariance matrix $R$. The most convenient way to present this information to the Kalman filter is in terms of one of the three-dimensional parameterizations of the deviation between the observed and predicted attitudes

$$
q_{o b s}=\delta q\left(\mathbf{x}_{\text {obs }}\right) \otimes \hat{q}(-) \text {. }
$$

The measurement model is simply

$$
\mathbf{h}(\mathbf{x})=\mathbf{x} \text {, }
$$

so $H=\partial \mathrm{h} / \partial \mathrm{x}$ is the $3 \times 3$ identity matrix and $R$ is the covariance of this error angle vector. The state update simplifies to

$$
\hat{\mathbf{x}}(+)=\hat{\mathbf{x}}(-)+P_{3 \times 3}(-)\left[P_{3 \times 3}(-)+R\right]^{-1}\left[\mathbf{x}_{o b s}-\hat{\mathbf{x}}(-)\right] .
$$

It is important to use the same three-dimensional parameterization in the observation processing, Eq. (81), as is used in the reset, Eq. (80). With this proviso, we see that when $R \ll P_{3 \times 3}(-)$, so that $\hat{\mathbf{x}}(+)=\mathbf{x}_{\text {obs }}$, the reset quaternion estimate is $\hat{q}(+)=q_{\text {obs }}$.

Projections in quaternion space can be used to map a vector measurement into a linear quaternion measurement [40]. This mapping is based on the observation that the quaternion using the minimumangle rotation to map a reference vector $r$ into the body frame vector $\mathbf{b}$ is

$$
q_{1} \equiv \frac{1}{\sqrt{2(1+\mathbf{b} \cdot \mathbf{r})}}\left[\begin{array}{c}
\mathbf{b} \times \mathbf{r} \\
1+\mathbf{b} \cdot \mathbf{r}
\end{array}\right] .
$$

The most general quaternion that maps $\mathbf{r}_{i}$ into $\mathbf{b}_{i}$ is

$$
\begin{aligned}
q & =\left[\begin{array}{c}
\mathbf{b} \sin \left(\vartheta_{b} / 2\right) \\
\cos \left(\vartheta_{b} / 2\right)
\end{array}\right] \otimes q_{1} \otimes\left[\begin{array}{c}
\mathbf{r} \sin \left(\vartheta_{r} / 2\right) \\
\cos \left(\vartheta_{r} / 2\right)
\end{array}\right] \\
& =[\cos (\vartheta / 2)] q_{1}+[\sin (\vartheta / 2)] q_{2},
\end{aligned}
$$


where $\vartheta_{b}$ and $\vartheta_{r}$ are arbitrary angles of rotation about $\mathbf{b}$ and $\mathbf{r}$, respectively, $\vartheta=\vartheta_{b}+\vartheta_{r}$, and

$\left.q_{2} \equiv \frac{1}{\sqrt{2(1+\mathbf{b} \cdot \mathbf{r})}} \mid \begin{array}{c}\mathbf{b}+\mathbf{r} \\ 0\end{array}\right]$.

The quaternion $q_{2}$ maps $\mathbf{r}$ into $\mathrm{b}$ by means of a $180^{\circ}$ rotation about the bisector of $\mathbf{b}$ and $\mathbf{r}$.

Equation (85) expresses $q$ as a linear combination of the two orthogonal quaternions $q_{1}$ and $q_{2}$, which constitute an orthogonal basis for the two-dimensional subspace of four-dimensional quaternion space consistent with the measurement. The four quaternions $q_{1}, q_{2}$,

$q_{3} \equiv \frac{1}{\sqrt{2(1-\mathbf{b} \cdot \mathbf{r})}}\left[\begin{array}{c}-(\mathbf{b} \times \mathbf{r}) \\ 1-\mathbf{b} \cdot \mathbf{r}\end{array}\right]$,

and

$q_{4} \equiv \frac{1}{\sqrt{2(1-\mathbf{b} \cdot \mathbf{r})}}\left[\begin{array}{c}\mathbf{b}-\mathbf{r} \\ 0\end{array}\right]$

constitute a complete orthogonal basis for fourdimensional quaternion space. Our linear measurement is created by noting that the true quaternion must be in the subspace spanned by $q_{1}$ and $q_{2}$, so it orthogonal to $q_{3}$ and $q_{4}$. This means that we can define a two-component measurement vector $\chi$ obeying the equation

$\chi \equiv\left[q_{3} q_{4}\right]^{T} q=$ white measurement noise .

This measurement model has been employed in an attitude filter with guaranteed convergence [41].

\section{Sequential Methods Related to Wahba's Problem}

We have seen that the EKF estimates deviations from a nominal attitude, so some a priori attitude estimate is required. Single-frame solutions of Wahba's problem do not require an a priori attitude estimate. We now discuss attempts to combine the best features of these two approaches [42-44].

\section{Filter QUEST}

The nine components of the 'attitude profile matrix' $B$, defined by Eq. (5), contain full information about the attitude (with three degrees of freedom) and the angular error covariance (with six independent components), so the EKF could be implemented on this matrix $[5,42]$. This provides little computational advantage, though, so Shuster proposed a simpler Filter QUEST algorithm, based on propagating and updating $B$ :
$B\left(t_{k}\right)=\mu \Phi_{3 \times 3}\left(t_{k}, t_{k-1}\right) B\left(t_{k-1}\right)+\sum_{t} a_{l} \mathbf{b}_{\imath} \mathbf{r}_{i}^{T}$

where $\Phi_{3 \times 3}\left(t_{k}, t_{k-1}\right)$ is the error state transition matrix of $\mathrm{Eq}$. (70) and the sum is over observations at time $t_{k}$. The "fading memory" factor, $\mu<1$, approximates the effect of the process noise. Any algorithm for solving Wahba's problem can estimate the attitude from $B\left(t_{k}\right)$.

\section{Recursive QUEST (REQUEST)}

This alternative sequential algorithm [43] propagates and updates Davenport's $K$ matrix by

$$
K\left(t_{k}\right)=\mu \Phi_{4 \times 4}\left(t_{k}, t_{k-1}\right) K\left(t_{k-1}\right) \Phi_{4 \times 4}^{\mathrm{T}}\left(t_{k}, t_{k-1}\right)+\sum_{i} a_{i} K_{i},
$$

where $\Phi_{4 \times 4}\left(t_{k}, t_{k-1}\right)$ is the quatemion state transition matrix of Eq. (56) and $K_{i}$ is the Davenport matrix for a single observation,

$$
K_{i} \equiv\left[\begin{array}{cc}
\mathbf{b}_{i} \mathbf{r}_{i}^{\mathrm{T}}+\mathbf{r}_{i} \mathbf{b}_{i}^{\mathrm{T}}-\left(\mathbf{b}_{i} \cdot \mathbf{r}_{i}\right) I & \left(\mathbf{b}_{i} \times \mathbf{r}_{i}\right) \\
\left(\mathbf{b}_{t} \times \mathbf{r}_{i}\right)^{\mathrm{T}} & \mathbf{b}_{i} \cdot \mathbf{r}_{i}
\end{array}\right] .
$$

Filter QUEST and REQUEST are mathematically equivalent, but Filter QUEST requires fewer computations. Neither has been competitive with the EKF in practice, largely due to the suboptimality of the fading memory approximation of the process noise. Computing the fading memory factor by a Kalmangain-like algorithm gives better performance, but sacrifices much of the attractive simplicity of these methods [44].

\section{SUMMARY}

Attitude determination is a crucial part of every space mission that employs actively controlled spacecraft or collects data whose interpretation depends on the orientation of the spacecraft. This includes almost every space mission. Attitude accuracy requirements vary dramatically, from tens of degrees to hundredths of arcsecond, covering a range of more than six orders of magnitude. Attitude determination for attitude control must be performed onboard the spacecraft, of course; but attitude determination for scientific data analysis can be performed onboard, on the ground, or split between the two places. Dramatic decreases in the price, weight, and power of space-hardened microprocessors has moved the center of gravity of attitude determination toward the spacecraft. Some 'smart' sensors, particularly star trackers, use embedded microprocessors, databases, and computational algorithms to compute attitude autonomously. Much of the techniques used for attitude determination are classical, but the field never ceases to advance. 


\section{REFERENCES}

1. Wertz, James R., ed., Spacecraft Attitude Determination and Control, Dordrecht, Holland, D. Reidel, 1978.

2. Markley, F. Landis, "Optimal Attitude Matrix from Two Vector Measurements," Journal of Guidance, Control, and Dynamics, Vol. 31, No. 3, pp. 765$768,2008$.

3. Black, Harold D., "A Passive System for Determining the Attitude of a Satellite," AIAA Journal, Vol. 2, No. 7, pp. 1350-1351, 1964.

4. Wahba, Grace, "A Least Squares Estimate of Spacecraft Attitude," SIAM Review, Vol. 7, No. 3, p. $409,1965$.

5. Shuster, Malcolm D., "Maximum Likelihood Estimate of Spacecraft Attitude," The Journal of the Astronautical Sciences, Vol. 37, No. 1, pp.7988, 1989.

6. Farrell, J. L., and J. C. Stuelpnagel; Wessner, R. H.; Velman, J. R.; and Brock, J. E.; "A Least Squares Estimate of Spacecraft Attitude," SIAM Review, Vol. 8, No. 3, pp. 384-386, 1966.

7. Horn, Roger A. and Charles R. Johnson, Matrix Analysis, Cambridge, UK, Cambridge University Press, 1985.

8. Davenport, Paul D., "A Vector Approach to the Algebra of Rotations with Applications," NASA Technical Note NASA TN D-4696, Greenbelt, MD, August 1968.

9. Keat, J., "Analysis of Least-Squares Attitude Determination Routine DOAOP," Computer Sciences Corporation Report CSC/TM-77/6034, February 1977.

10. Golub, Gene H. and Charles F. Van Loan, Matrix Computations, Baltimore, MD, The Johns Hopkins University Press, 1983.

11. Shuster, M. D. and S. D. Oh, "Three-Axis Attitude Determination from Vector Observations," Journal of Guidance and Control, Vol. 4, No. 1, pp. 70-77, 1981.

12. Shuster, Malcolm D. and Gregory A. Natanson, "Quaternion Computation from a Geometric Point of View," The Journal of the Astronautical Sciences, Vol. 41, No. 4, pp. 545-556, 1993.
13. Cheng, Y., and Shuster, M. D., "Robustness and Accuracy of the QUEST AIgorithm," in Spaceflight Mechanics 2007, Advances in the Astronautical Sciences, Vol. 127, pp. 41-62, San Diego, CA: Univelt, Inc. 2007. (AAS/AIAA Space Flight Mechanics Meeting, Sedona, AZ, 2007).

14. Markley, F. Landis, "Attitude Determination Using Vector Observations and the Singular Value Decomposition," The Journal of the Astronautical Sciences, Vol. 36, No. 3, pp. 245-258, 1988.

15. Markley, F. Landis, "Attitude Determination Using Vector Observations: a Fast Optimal Matrix Algorithm," The Journal of the Astronautical Sciences, Vol. 41, No. 2, pp. 261-280, 1993.

16. Mortari, Daniele, "ESOQ: A Closed-Form Solution to the Wahba Problem," The Journal of the Astronautical Sciences, Vol. 45, No. 2, pp. 195204, 1997.

17. Mortari, Daniele, " $n$-Dimensional Cross Product and its Application to Matrix Eigenanalysis," Journal of Guidance, Control, and Dynamics, Vol. 20, No. 3, pp. 509-515, 1997.

18. Mortari, Daniele, "Second Estimator of the Optimal Quaternion," Journal of Guidance. Control, and Dynamics, Vol. 23, No. 5, pp. 885$888,2000$.

19. Markley, F. Landis, and Daniele Mortari, "Quaternion Attitude Estimation Using Vector Observations," The Journal of the Astronautical Sciences, Vol. 28, No. 2/3, pp. 359-380, 2000.

20. Cheng, Y., and Shuster, M. D., "The Speed of Attitude Estimation," in Spaceflight Mechanics 2007, Advances in the Astronautical Sciences, Vol. 127, pp. 101-116, San Diego, CA: Univelt, Inc. 2007. (AAS/AIAA Space Flight Mechanics Meeting, Sedona, AZ, 2007).

21. Crassidis, J. L., Markley, F. L., and Lightsey, E. G., "Global Positioning System Integer Ambiguity Resolution Without Attitude Knowledge," Journal of Guidance, Control. and Dynamics, Vol. 22, No. 2, pp. 212-218, 1999.

22. Psiaki, M. L., and Mohiuddin, S., "Global Positioning System Integer Ambiguity Resolution Using Factorized Least-Squares Techniques," Journal of Guidance, Control, and Dynamics, Vol. 30, No. 2, pp. 346-356, 2007. 
23. Cohen, C. E., "Attitude Determination," in Global Positioning System: Theory and Applications,

Volume 2, ed. By B. W. Parkinson and J. J. Spilker, Progress in Aeronautics and Astronautics, Vol. 164, Washington, DC, American Institute of Aeronautics and Astronautics, 1996.

24. Crassidis, John L., and F. Landis Markley, "New Algorithm for Attitude Determination Using Global Positioning System Signals," Journal of Guidance, Control, and Dynamics, Vol. 20, No. 5, pp. 891-896, 1997.

25. Bar-Itzhack, Itzhack Y., Paul Y. Montgomery, and Joseph C. Garrick, "Algorithm for Attitude Determination Using the Global Positioning System," Journal of Guidance, Control, and Dynamics, Vol. 21, No. 6, pp. 846-852, 1998.

26. Hashmall, J., K. Liu, and M. Rokni, "Accurate Spacecraft Attitudes from Magnetometer Data," CNES Spaceflight Dynamics (Colloque 95), Toulouse, France, Cépaduès-Éditions, 1995.

27. Kalman, R. E., "A New Approach to Linear Filtering and Prediction Problems," Transactions of the ASME, Series D, Journal of Basic Engineering, Vol. 82, pp. 35-45, 1960.

28. Gelb, Arthur, ed., Applied Optimal Estimation, Cambridge, MA, the MIT Press, 1974.

29. Crassidis, J. L., and Junkins, J. L., Optimal Estimation of Dynamic Systems, Chapman \& Hall/CRC, Boca Raton, FL, 2004.

30. Lefferts, E. J., F. L. Markley, and M. D. Shuster, "Kalman Filtering for Spacecraft Attitude Estimation," Journal of Guidance, Control, and Dynamics, Vol. 5, No. 5, pp. 417-429, 1982.

31. Bar-Itzhack, I. Y., J. Deutschmann, and F. L. Markley, "Quaternion Normalization in Additive EKF for Spacecraft Attitude Determination," AIAA Paper 91-2706, AIAA Guidance, Navigation, and Control Conference, New Orleans, LA, August 1991.

32. Deutschmann, J., F. L. Markley, and I. Bar-Itzhack, "Quaternion Normalization in Spacecraft Attitude Determination," Flight Mechanics/Estimation Theory Symposium, NASA Conference Publication 3186, Goddard Space Flight Center, Greenbelt, MD, May 1992
33. Markley, F. L., "Attitude Filtering on $\mathrm{SO}(3)$," The Journal of the Astronautical Sciences, Vol 54, No. 3/4, pp. 391-413 (2006)

34. Toda, N. F., J. L. Heiss, and F. H. Schlee, "SPARS: the System, Algorithm, and Test Results," Proceedings of the Symposium on Spacecraft Attitude Determination, Aerospace Corp. Report TR-0066 (5306)-12, Vol. 1, pp. 361-370, Sept.Oct. 1969.

35. Murrell, James W., "Precision Attitude Determination for Multimission Spacecraft," AIAA Paper 78-1248, AlAA Guidance and Control Conference, Palo Alto, CA, August 1978.

36. Markley, F. L., "Attitude Error Representations for Kalman Filtering," Journal of Guidance, Control, and Dynamics, Vol. 26, No. 2, pp. 311-317, 2003.

37. Stuelpnagel, John, "On the Parameterization of the Three-Dimensional Rotation Group," SIAM Review, Vol. 6, No. 4, pp. 422-430, 1964.

38. Shuster, Malcolm D., "A Survey of Attitude Representations," The Journal of the Astronautical Sciences, Vol. 41, No. 4, pp. 439-517, 1993.

39. Shuster, M. D., "Kalman Filtering of Spacecraft Attitude and the QUEST Model," The Journal of the Astronautical Sciences, Vol. 38, No. 3, pp. 377-393, 1990.

40. Reynolds, R. G., "Quaternion Parameterization and a Simple Algorithm for Global Attitude Estimation," Journal of Guidance, Control, and Dynamics, Vol. 21, No. 4, pp. 669-671, 1998.

41. Reynolds, R. G., "Asymptotically Optimal Attitude Filtering with Guaranteed Convergence," Journal of Guidance, Control, and Dynamics, Vol. 31, No. 1, pp. 114-122, 2008.

42. Shuster, Malcolm D., "A Simple Kalman Filter and Smoother for Spacecraft Attitude," The Journal of the Astronautical Sciences, Vol. 37, No. 1,pp. 89-106, 1989.

43. Bar-Itzhack, I. Y., "REQUEST: A Recursive QUEST Algorithm for Sequential Attitude Determination," Journal of Guidance, Control, and Dynamics, Vol. 19, No. 5, pp. 1034-1038, 1996.

44. Choukroun, D., Bar-Itzhack, I. Y., and Oshman, Y., "Optimal-REQUEST Algorithm for Attitude Determination," Journal of Guidance, Control, and Dynamics, Vol. 27, No. 3, pp. 418-425, 2000. 